A N N A L E S Annales de Bretagne et des Pays de l'Ouest

\title{
Un héroïsme républicain
}

L'exemple du sauveteur dans Le Nouvelliste du Morbihan (1887-1914)

Republican heroism. The example of the rescuer in Le Nouvelliste du Morbihan (1887-1914)

\section{Anne Schmitt et Jean-Nicolas Renaud}

\section{OpenEdition \\ Journals}

Édition électronique

URL : http://journals.openedition.org/abpo/3064

DOI : $10.4000 / a b p o .3064$

ISBN : 978-2-7535-4275-4

ISSN : 2108-6443

Éditeur

Presses universitaires de Rennes

\section{Édition imprimée}

Date de publication : 30 juin 2015

Pagination : 99-117

ISBN : 978-2-7535-4273-0

ISSN : 0399-0826

Référence électronique

Anne Schmitt et Jean-Nicolas Renaud, "Un héroïsme républicain », Annales de Bretagne et des Pays de I'Ouest [En ligne], 122-2 | 2015, mis en ligne le 30 juin 2017, consulté le 01 mai 2019. URL : http:// journals.openedition.org/abpo/3064; DOI : 10.4000/abpo.3064 


\title{
Un héroïsme républicain L'exemple du sauveteur dans Le Nouvelliste du Morbihan (1887-1914)
}

\author{
Anne SCHMITT \\ Élève normalienne, ENS-Rennes \\ Jean-Nicolas RENAUD \\ Maître de conférences en histoire du sport, ENS-Rennes, laboratoire VIP\&S (EA463)
}

\begin{abstract}
«Une petite fille de 4 ans, Fernande Mercier, s'amusait samedi soir vers 5 heures à courir sur le parapet du quai Rohan, le long du grand trou qui existe à cet endroit. Brusquement elle tomba dans l'eau. Un citoyen courageux, Allain Jacques, chaudronnier au Manio, se précipita au secours de l'enfant qu'il ramena sur le bord et qui fut ensuite reconduit chez son oncle M. Faucheux, demeurant rue de la Comédie 12."
\end{abstract}

Ce fait divers, relaté dans Le Nouvelliste du Morbihan, le 16 septembre 1897, rapporte avec précision le sauvetage d'une jeune enfant. Cet exemple mêle, dans une véritable mise en scène, héroïsme extraordinaire et citoyenneté quotidienne. La presse morbihannaise évoque fréquemment ces incidents. Jouant de ses atouts journalistiques, le chroniqueur accentue le sentiment de détresse de la victime et, par effet de contraste, auréole d'un grand courage le geste gratuit de ce secouriste improvisé.

Cette figure du sauveteur s'inscrit dans une Bretagne de la fin du $\mathrm{XIX}^{\mathrm{e}}$ siècle qui est en pleine reconstruction économique et dans une France soumise aux idées de solidarisme et au principe d'obligation morale de Léon Bourgeois ${ }^{1}$. L'activité portuaire de Lorient croît. La cité investit dans le domaine de la pêche comme de la marine que des échanges avec la Grande-Bretagne, en cette période de détente, accentuent. La rapide évolution technologique des bateaux assoit plus encore la relation existant entre économie et océan. La préfecture maritime morbihannaise conforte ainsi son statut de ville-port. Moyen de vivre et de prospérer, le milieu

1. JEANNESSON, Stanislas, "Léon Bourgeois aux conférences de La Haye de 1899 et 1907 : solidarisme et démocratisation des relations internationales ", Histoire, économie et société, 2/2014 (33e année), p. 107-120. 
marin fascine les Bretons par sa puissance et ses mystères, inspirant contes et légendes. Mais hostile, imprévisible et regorgeant de dangers mortels auxquels les Lorientais doivent faire face quotidiennement, il effraye également; une " mer nécessaire autant que redoutable ${ }^{2}$ ". Dans le sonnet Oceano nox ${ }^{3}$, Victor Hugo convoque les masses anonymes qui hantent les flots sombres autant que l'imaginaire social. Le romantisme affecté de l'auteur renvoie également, dans Notre-Dame de Paris ${ }^{4}$, à la notion de fatalité. Or, la fin du XIX ${ }^{\mathrm{e}}$ siècle devient le théâtre d'un nouveau rapport à l'homme. Avec les idées des Lumières et de la Révolution incarnées dans une III ${ }^{\mathrm{e}}$ République qui prend ses distances avec les valeurs chrétiennes, l'individu possède le choix nouveau d'infléchir son destin ${ }^{5}$. La grève, plus encore que la haute mer, représente une frontière physique et symbolique où flue et reflue le jeu de la vie et de la mort.

À travers le prisme de cette fatalité qu'incarne l'océan pour les habitants d'une ville portuaire, le statut du sauveteur dans la presse prend une dimension particulière. Il est l'espoir face au sort implacable que peut représenter le piège océan. Construite à partir des récits de naufrage, la représentation de la mort par noyade reste associée à l'absence de corps sur lequel faire son deuil au plan social, auquel administrer les derniers sacrements au plan spirituel ${ }^{6}$. Dans l'espace lorientais comme sur le territoire de la Bretagne en crise, le sauveteur incarne un renouveau de l'Homme et en cela du Breton, une victoire du citoyen sur le destin - funeste - tracé par Dieu. À l'époque, la presse connaît son âge d'or ${ }^{7}$. Le chroniqueur, porté par un lectorat en expansion, agit sur les représentations sociales locales et, en l'occurrence, construit une valorisation d'un ordre moral, un devoir moral au sens de Léon Bourgeois, qui prend corps dans l'acte du sauveteur; une obligation comme contrepartie de ce pouvoir nouveau acquis par l'homme sur sa destinée. Par effet ricochet, cette démarche aboutit à la composition d'un héroïsme autour du citoyen secoureur ${ }^{8}$. Mais dans la période qui précède la Grande Guerre, il est décrit de deux manières distinctes : tantôt " improvisé ", tantôt " professionnel ". La figure varie et la mise en scène changent de concert. Pourtant, celui qui sauve une vie, quel que soit son statut, devrait pouvoir bénéficier de la même considération. La différenciation dans leur traitement semble se faire sur une valeur à la symbolique forte : l'altruisme spontané.

2. LECOCQ, Danielle, " De l'eau primitive à l'océan infranchissable », dans CoRBIN, Alain et RichaRD, Hélène (dir.), La mer, terreur et fascination, Paris, Seuil, 2004, p. 44.

3. Hugo, Victor, Les rayons et les ombres, 1840.

4. Hugo, Victor, Notre-Dame de Paris, Paris, Hachette, 1858.

5. Foucault, Michel, «Qu'est-ce que les Lumières? » dans Dits et Écrits, Paris, Gallimard, tome IV, 1994, p. 562-578.

6. CABantous, Alain, "Fortunes de mer ", dans Corbin, Alain et RichaRd, Hélène (dir.), La mer..., op. cit., p. 130.

7. ALBERT, Pierre, Histoire de la presse, Paris, PUF, 2010, p. 64.

8. Nous distinguons le secoureur du secouriste dans le sens où ce dernier renvoie à un technicien habilité et non à un individu quelconque secourant quelqu'un sans préméditation. 
Le corpus que nous utilisons pour analyser la construction de l'héroïsme autour du citoyen secoureur comprend 178 actes de sauvetages relevés sur 27 ans de publications rapportées dans Le Nouvelliste du Morbihan $^{9}$. Ce travail vise à caractériser la façon dont ces actes sont relatés afin d'identifier les valeurs, et la morale sous-jacente, qui conduisent à la composition d'un héroïsme propre à cette époque. La particularité de l'étude réside dans le croisement de deux regards, attenants à une même source. L'image construite du sauveteur improvisé se confronte à celle élaborée du professionnel.

L'acte de dévouement constitue la pierre angulaire du discours journalistique en ce qu'il représente une exposition à la mort à la fois volontaire et paradoxalement imprévue du sauveteur pour arracher la victime à un trépas annoncé. Ce geste fonde les différences de traitement du chroniqueur car il distingue les contextes de sauvetage et, de facto, le statut du sauveteur. Le professionnel ne risque pas grand-chose; son investissement est louable, mais normal. Le sauveteur improvisé fait don de lui au nom du bien suprême que constitue la vie ${ }^{10}$. Mais au-delà de ce point s'agence tout un ensemble d'énoncés qui élève l'homme au rang d'un héroïque sauveteur ou le maintient dans son statut d'honnête travailleur. Il existerait donc un devoir moral qui conduirait le quidam à prendre des risques pour sauver la vie d'un concitoyen au nom de cette seule relation d'appartenance à une communauté nationale. La protection mutuelle au sein du collectif se doit d'être tellement intégrée aux comportements de chacun qu'elle relève d'un réflexe, et d'un peu de courage. Elle ne saurait être réfléchie sans sembler être intéressée. C'est sur la récupération par la presse locale de cette pulsion à des fins de diffusion d'un « imaginaire normatif commun ${ }^{11}$ " que cette étude, finalement, se penche ${ }^{12}$.

\section{Le Breton et la mer...}

À la fin du XIX ${ }^{\mathrm{e}}$ siècle, la Bretagne sort d'une crise économique et sanitaire qui a marqué les Bretons sur plus de cent ans. La misère, extrêmement présente dans les zones rurales, conjugue une propagation plus forte de la maladie et un manque important de travail ${ }^{13}$. Les habitants de la campagne

9. Étude portant sur six années $(1887,1892,1897,1903,1908,1914)$ allant du mois de mai au mois de septembre, c'est-à-dire sur les mois comprenant les périodes de bain et justifiant, sur la période, la comparaison du sauveteur professionnel et du sauveteur improvisé. Un tableau récapitulatif de la répartition des articles en fonction du sauveteur concerné est présent en fin d'article.

10. ARENDT, Hannah, Condition de l'homme moderne, Paris, Calmann-Lévy, 1983, p. 390.

11. KALIFA, Dominique et RÉGNIER, Philippe, "Homogénéiser le corps national ", dans KaLIFA Dominique et al., La civilisation du journal. Histoire culturelle et littéraire de la presse française au XIX siècle, Nouveau Monde Édition, 2011, p. 1419.

12. Le processus serait certainement lisible à travers des articles de presse mentionnant des actes d'héroïsme. Nous nous sommes restreints au sauvetage, symboliquement plus prégnant dans l'espace portuaire de Lorient.

13. CASSARD, Jean-Christophe et MoniER, Jean-Jacques, Toute l'histoire de la Bretagne des origines à nos jours, Morlaix, Skol Vreizh, 2012. 
migrent donc dans les zones urbaines à la recherche d'une activité professionnelle et d'une vie meilleure ${ }^{14}$. Dans ce contexte, le port de Lorient s'industrialise et se développe, notamment grâce à l'importation du charbon, de la construction navale et surtout à l'instauration d'une pêche dotée de chalutiers à vapeur ${ }^{15}$. Une course à l'équipement entre les ports du grand Ouest, comme Brest et Saint-Nazaire, s'engage. La concurrence fait rage. Le rapport à l'océan s'avère extrêmement utilitaire. Or, le développement de l'activité et les évolutions des équipements contribuent à l'amélioration de la vie des pêcheurs bretons. Les salaires augmentent. La sécurité s'accroît avec l'apport de moyens de secours ${ }^{16}$, même sommaires. Mais, entre 1902 et 1913, la pénurie de sardines au large des côtes atlantiques ${ }^{17}$ perturbe le secteur. Malgré d'appréciables changements, les conditions d'existence des pêcheurs restent précaires et de violentes manifestations éclatent ${ }^{18}$. L'océan reste une ressource instable et capricieuse, qui influe directement sur les habitants du littoral. Une part importante de la société locale en est totalement dépendante.

Un autre usage de la mer apparaît à cette époque. Tout au long du $\mathrm{XIX}^{\mathrm{e}}$ siècle, la concession de l'estran aux communes permet le développement du tourisme balnéaire sur les côtes morbihannaises ${ }^{19}$. À Port-Louis et Larmor-Plage, les bourgeois et nobles en villégiature profitent, de plus en plus nombreux, des bains de mer, dont les bienfaits sont loués par les médecins dès la seconde moitié du siècle ${ }^{20}$. Le phénomène du " tourisme balnéaire " prend de plus en plus d'ampleur; un rapport hédonique avec l'océan se construit dans les esprits bretons comme étrangers ${ }^{21}$. "Le XIX ${ }^{\mathrm{e}}$ siècle est celui de l'intégration des mondes littoraux dans la communauté nationale, c'est la rencontre entre ceux du rivage et ceux de l'inté-

14. Chedeville, André et Croix, Alain, Histoire de la Bretagne, Paris, PUF, 1993.

15. Chaumiel Louis, "Abrégé d'histoire de Lorient de la fondation (1666) à nos jours (1939) ", Annales de Bretagne et des pays de l'Ouest, 1939, Tome 46, numéro 1-2, p. 66-87.

16. Coulıou, Jean-René, "Souvenir et contraintes du temps qui passe pour les pêcheurs contemporains de la Bretagne méridionale ", Annales de Bretagne et des pays de l'Ouest, 2010, Tome 117, numéro 3, p. 147-159.

17. Fichou, Jean-Christophe, "La crise sardinière de 1902-1913 au cœur des affrontements religieux en Bretagne ", Annales de Bretagne et des pays de l'Ouest, 2009, Tome 116, numéro 4, p. 149-170.

18. LE BouËDEC, Gérard, "Le temps des crises dans les sociétés littorales (XVI ${ }^{\mathrm{e}}$ XIX ${ }^{\mathrm{e}}$ siècles) ", Annales de Bretagne et des pays de l'Ouest, 2010, Tome 117, numéro 3, p. 14-24. En 1894, 69\% des travailleurs lorientais sont embauchés dans les trois conserveries de sardines (CORNETTE, Joël, Histoire de la Bretagne et des Bretons, Paris, Seuil, Tome 2, 2005, p. 327).

19. LE BOUËDEC, Gérard, " Pour une histoire de l'estran français du XVI ${ }^{\mathrm{e}}$ siècle à la Seconde Guerre mondiale ", Annales de Bretagne et des pays de l'Ouest, Tome 117, numéro 4, 2010 , p. 136-164.

20. TERRET, Thierry, "Bains de mer du Nord et natation au xIX ${ }^{\mathrm{e}}$ siècle : pratiques hygiéniques et loisirs de classes ", Sport-Histoire, 1988, n² 2, Toulouse, Privat, p. 9-22.

21. Clairay, Philippe et VincENT, Johan, " Le développement balnéaire breton : une histoire originale ", Annales de Bretagne et des pays de l'Ouest, 2008, Tome 115, numéro 4, p. 201-233. 
rieur $^{22}$ ". Le bord de mer demeure cependant une frontière, une zone de cisaillement où l'homme est en danger ${ }^{23}$. Ce phénomène implique une organisation nouvelle des villes du littoral qui évoluent et se transforment au rythme de l'alternance des périodes estivales et des périodes où la vie touristique est en veille ${ }^{24}$. L'océan utilitaire des habitants du littoral, qui récupèrent le goémon pour le brûler et raclent les plages pour récupérer le sable, cohabite difficilement avec la quête d'un milieu paisible et paradoxalement sauvage de touristes en quête d'exotisme aseptisé. Toutefois, cette activité, symbole d'un renouveau économique impliquant la balnéarisation des côtes, devient peu à peu plus lucratif que le cabotage ou la pêche ${ }^{25}$. En même temps, la pratique du loisir renforce la place accordée à cet espace intermédiaire, usage prolongé d'un lieu naguère de passage, entre la rive et la mer.

Si les Lorientais placent le rapport à l'océan au cœur de leurs activités, la noyade demeure l'une des morts violentes les plus répandues et les plus effrayantes. Face à cette force de la nature, les Travailleurs de la mer sont impuissants et en perpétuel danger sur leur lieu professionnel. Le sauvetage représenterait alors une victoire de l'homme sur la fatalité, dont la presse se ferait l'écho parce que le média se nourrit de tout événement quotidien capable de susciter l'intérêt de son lectorat. La réalité de la vie, telle qu'elle est décrite dans les colonnes du Nouvelliste du Morbihan, place l'histoire sur un fil entre distance et proximité. Le feuillet relate ce qui peut arriver à tout le monde mais qui, en l'occurrence, atteint un autrui distancié.

Entre 1887 et 1914, l'essor du Nouvelliste du Morbihan semble bénéficier de la croissance économique de Lorient ${ }^{26}$. Le journal, acheté par les frères Alexandre et Charles Cathrine en 1886, est tiré à 1000 exemplaires à ses débuts, puis à 10000 en 1893 et 60000 en 1904 jusqu'à devenir le journal le plus important de la région ${ }^{27}$. La proximité des habitants avec l'océan, les noyades qui frappent aveuglément ${ }^{28}$ et le goût croissant pour ces faits divers expliqueraient la présence régulière des actes de sauvetage au sein de cette presse locale. À l'image de la région, le journal investit, progressivement tout au long de notre période, la morale républicaine ${ }^{29}$ sans brusquer les valeurs chrétiennes d'altruisme fortement ancrées en Bretagne. Cependant, si le ver-

22. LE BOUËDEC, Gérard, "Le temps des crises... ", op. cit., p. 15.

23. On se réfère à l'idée qu'il existe trois espaces : le bord de mer, les rouleaux et la haute mer selon HARTOG, François " La haine de Poséïdon ", dans CORBIN, Alain et RICHARD, Hélène (dir.), La mer..., op. cit., p. 89.

24. VINCENT, Johan, "Hors saison : une saison à préparer la station balnéaire (Bretagnesud et Vendée, 1850-1945)", Annales de Bretagne et des pays de l'Ouest, 2010, Tome 117, numéro 3, p. 114-126.

25. Le BouËDEC, Gérard, "Pour une histoire sociale de l'estran français ...", op. cit.

26. Van Meeuwen, Gil, L'aventure du Nouvelliste du Morbihan. Du journal à l'écran, mémoire de maîtrise, Université Bretagne-Sud, 2001.

27. Bluysen, Paul, Annuaire de la presse française et étrangère, Paris, 1909, p. 565.

28. ChESNAIS, Jean-Claude, "Les morts violentes en France depuis 1826 : comparaison internationale ", Vol. 75, Ined, 1976.

29. CoRnetTE, Joël, Histoire de la Bretagne..., op. cit., p. 385. 
sant religieux s'était avéré plus prégnant pour ce journal bihebdomadaire, la recherche d'un homme d'Église pour administrer les derniers sacrements au mourant aurait plus régulièrement accompagné celle d'un homme de médecine visant à rendre ces derniers superflus. Or, ce n'est pas le cas.

Par ailleurs, " [la] morbidité prend une place grandissante dans la "civilisation du journal" à partir des années 1880. La tendance est générale et se retrouve dans tous les périodiques. Aussi, tout ce qui se rapporte à la mort, aux cadavres, aux accidents de toutes sortes fait partie de l'univers journalistique et correspond à l'horizon d'attente du lecteur ${ }^{30}$ ". Si les cas de suicide trouvent leur place dans les feuillets, les noyades et les actes de sauvetages font également partie du contenu informatif dont les lecteurs sont friands ${ }^{31}$. Les narrations de ces instants où la vie est en suspens relèvent d'un double niveau de discours. D'une part, elles illustrent le quotidien de chaque Lorientais et participent à l'élaboration d'une communauté de vie, synonyme de valeurs partagées ${ }^{32}$; de l'autre, elles donnent à lire une gestion politique de l'espace local. Les côtes et le port deviennent le théâtre d'actes de courage et de dévouement, qui font le bonheur des chroniqueurs en constante recherche de sensationnel. Suspense, mort en sursis, héroïsme; dans cet univers connu sourdent les nombreux ingrédients capables de piquer la curiosité et de caresser l'humanisme désintéressé de tous. Entre misère anecdotique et illustration affective d'un phénomène social, le sauvetage de bord de mer tel qu'il est donné à voir dans l'articulet fusionne le réel et le mythique. Le courageux imprudent qui se jette dans le sombre bouillon relève du statut de l'Homme providentiel ${ }^{33}$ et, à ce titre, teinte plus encore de réalisme la narration du fait héroïque.

Le journal se veut l'émanation de la vox populi et l'incarnation de ses valeurs. À ce titre, il vient couvrir tout bienfaiteur d'un honneur populaire en complément d'une hypothétique distinction par les édiles, par laquelle l'État reconnaît officiellement la portée sociétale du geste :

" Il y a acte de courage et de dévouement lorsqu'une personne, pour en retirer une autre d'un péril imminent ou pour prévenir un accident qui pourrait avoir des conséquences graves expose sciemment et volontairement sa propre existence. Mais accomplir un simple acte d'humanité, apporter un zèle plus ou moins grand à s'acquitter d'un devoir, ce sont là des faits qui, quelques louables qu'ils soient, ne sauraient être considérés comme acte de dévouement. " (Circulaire ministérielle 5 avril 1858) ${ }^{34}$.

30. Glatigny, Jessica, " " Une anticipation de l'inéluctable, un raccourci définitif ". Le suicide dans la presse et dans l'Avenir de la Vienne en 1890 ", Annales de Bretagne et des Pays de l'Ouest, 2009, Tome 116, $\mathrm{n}^{\circ}$ 1, p. 57.

31. Chevalier, Louis, Splendeurs et misères du fait divers, Paris, Perrin, 2011, p. 37.

32. Ambroise-Rendu, Anne Claude, "Les faits divers ", dans Kalifa, Dominique et al., La civilisation du journal. Histoire culturelle et littéraire de la presse française au XIXe siècle, Paris, Nouveau Monde Édition, 2011, p. 979.

33. Girardet, Raoul, Mythes et mythologies politiques, Paris, Seuil, 1990, p. 77.

34. CAILLE, Frédéric, " La vertu en administration. La médaille de sauvetage, une signalétique officielle du mérite moral au xIX ${ }^{\mathrm{e}}$ siècle ", Genèses, vol. 28, n 1, 1997, p. 29-51. 
Pour mériter les honneurs et prétendre à un statut héroïque, l'acte de dévouement exige de risquer sa vie pour porter secours à autrui ${ }^{35}$. Cette base fonde l'héroïsme du sauveteur. De plus, un individu « ne devient réellement héroïque qu'à partir du moment où la foule a le sentiment de lui devoir quelque chose ${ }^{36} »$. On retrouve cet élément lorsque le journaliste remercie le sauveteur à la fin du récit de l'acte de sauvetage. Le chroniqueur incite les lecteurs à éprouver de la reconnaissance, à lui rendre les honneurs ${ }^{37}$ et transforme le héros de l'instant en une figure d'exemplarité anonyme que la République aime à accrocher au tableau des médailles ${ }^{38}$. La diffusion de ce modèle passe par la publication des comptes rendus de remise de récompense aux bienfaiteurs lorsque ceux-ci appartiennent à une société de sauvetage. La portée anthropologique de la dialectique du don et du contre-don ${ }^{39}$ génère un cycle infini d'obligations morales. Il se met en branle une valse à trois temps dans laquelle le geste instinctif valorise son glorieux auteur. Ce dernier reçoit les reconnaissances qui lui sont dues, lesquelles, en retour, obligent le héros, autant que ceux qui souhaitent l'imiter, à respecter cette image partagée de l'altruisme et de la solidarité. Qu'en est-il alors lorsque le valeureux quidam est isolé, au mauvais endroit, au bon moment? La presse joue alors un rôle de levier nouveau et, de facto, extrêmement efficient de diffusion d'une bravoure, condamnée à rester anonyme sans l'aide du journal.

Cette valorisation médiatique du sauveteur-citoyen apparaît nettement dans une société bretonne de plus en plus sensible aux idéaux républicains. Les actes de sauvetage donneraient ainsi corps aux valeurs citoyennes et républicaines. Ce type de situation en deviendrait le lieu d'expression par excellence $^{40}$. Leur présence dans les chroniques du Nouvelliste du Morbihan exhiberait une moralité en acte. Cette visibilité nouvelle du peuple, dans ce qu'il a de plus anonyme et de plus collectif, constitue une forme de réappropriation des symboles de souveraineté républicaine. Les fêtes civiques de Napoléon III, arguant de la remise de la médaille de Sainte-Hélène aux soldats survivants de son glorieux oncle, incarnaient un temps particulièrement fort d'imprégnation des mentalités ${ }^{41}$ aux valeurs de bravoure et

35. OLIVIER, Vincent, " Un sang-froid et un courage au-dessus de leur âge ", Annales de Bretagne et des pays de l'Ouest, Tome 116, $\mathrm{n}^{\circ}$ 1, 2009, p. 73-85.

36. Duret, Pascal, "Sémiotique de l'héroïsme sportif ", Revue STAPS, Vol. 13, n²9, 1991, p. 55-66.

37. HAZAREESINGH, Sudhir, « La légende napoléonienne sous le Second Empire : les médaillés de Sainte-Hélène et la fête du 15 août ", Revue Historique, 3/2003, n 627, p. 554.

38. IHL, Olivier, Le mérite et la République : essai sur la société des émules, Paris, NRF Gallimard, 2007.

39. MAuss, Marcel, «Essai sur le don. Forme et raison de l'échange dans les sociétés primitives ", L'année sociologique, 1924.

40. CAILle, Frédéric, «Le citoyen secoureur. Secours publics, sauveteurs et secouristes en France à la fin du XIX ${ }^{\mathrm{e}}$ siècle ", Politix, vol. 11, no 44, 1998, p. 39-50.

41. HAZAREESINGH, Sudhir, "L'opposition républicaine aux fêtes civiques du Second Empire : fête, anti-fête, et souveraineté ", Revue d'histoire du XIXe siècle, p. 149-171 [En ligne], 26/27, 2003, mis en ligne le 19 février 2008, consulté le 23 janvier 2015. 
d'honneur. Avec l'essor de la presse, des acteurs anonymes incarnent les qualités citoyennes, les rendant accessibles à tous et inversant le processus de valorisation sociale. Si naguère, l'autorité adoubait ses champions, la souveraineté du peuple devient, maintenant, visible à l'occasion de ces manifestations locales et quotidiennes, faisant de la République une réalité tangible et présente à tous les échelons de la vie sociale.

\section{Le sauvetage et les secours publics au xixe siècle}

Pour mieux comprendre le statut du sauveteur et notamment du sauveteur " professionnel ", un état des lieux des méthodes d'intervention et de secourisme semble nécessaire. Le développement de la baignade ludique et des activités en lien avec le milieu marin induit mécaniquement une augmentation des noyades, déjà fort fréquentes. Mais ces pratiques favorisent également l'apparition d'une natation plus utilitaire consacrée au sauvetage et à la surveillance des baigneurs ${ }^{42}$. L'instauration de Fédérations de Sauvetage et l'affectation par les municipalités d'un sauveteur avec un canot sur le lieu de baignade ${ }^{43}$ marquent une étape significative dans la gestion collective de ces accidents. Les sauveteurs occupant ce poste sont généralement de la région et familiers du milieu aquatique, incapables de dire quand et comment ils ont appris à nager; c'est pour eux quelque chose de naturel, aptitude couplée à un sens profond du dévouement.

Entre 1853 et 1903, 158 associations françaises de sauvetage et de sauveteurs ${ }^{44}$ sont déclarées. En 1865 est créée la Société Centrale de Sauvetage des Naufragés et, en 1873, la Société des Hospitaliers Sauveteurs Bretons ${ }^{45}$. Ces cercles portent assistance aux noyés et aux navires en difficulté, mais ont aussi la responsabilité des travaux maritimes les concernant (phares, bouées, balise, etc.). Paradoxalement, leur création relève d'intérêts économiques dans la mesure où leur mission principale consiste à secourir les biens en cas de naufrage ${ }^{46}$. De plus, les communes côtières encouragent et aident les sociétés de sauvetage en leur fournissant des locaux, un canot et des subventions. Néanmoins, leur multiplication ne compense pas le retard français en matière d'action publique à destination des secours en milieu maritime ou fluvial ${ }^{47}$. Elles marquent néanmoins un besoin devenu criant auprès des collectivités.

42. TERRET, Thierry, Naissance et diffusion de la natation sportive, Paris, L'Harmattan, 1994.

43. Ibidem.

44. CAILle, Frédéric, "Le citoyen secoureur... ", op. cit.

45. Ibidem.

46. Thibaudeau, Patricia, Sauver des vies : la société nationale de sauvetage en mer. Éditions Textuel, 2001.

47. VIVIER, Christian, La sociabilité canotière. La société nautique de Besançon, Paris, L'Harmattan, 1999. BRETin, Karen, "Avènement des sports nautiques à Chalon-sur-Saône (1877-1960) ", dans Movement et Sports sciences, Vol. 45, 2002, p. 67-84. 
Sortir la victime de l'eau lorsqu'elle est inconsciente ne suffit pas pour accomplir un acte complet de sauvetage et le manque de formation des sauveteurs en secourisme constitue une lacune réelle ${ }^{48}$. Les réflexions sur les secours à porter aux noyés commencent en France au XVIII siècle lorsque Philippe Nicolas Pia (1721-1799) met en place la première formation destinée aux militaires de la Garde de Paris ${ }^{49}$. À la fin du XIX ${ }^{\mathrm{e}}$ siècle, les techniques de réanimation sur le noyé sont multiples ${ }^{50}$; ces techniques sommaires cohabitent avec "l'incontournable " et redoutable saignée. Aussi, un congrès est organisé en 1879 à Paris afin de favoriser les échanges entre le monde médical et le monde du secourisme, mais aussi entre associations de sauvetage ${ }^{51}$. Le geste à réaliser pour sauver se rationalise et se diffuse par l'intermédiaire de ces sociétés. Au-delà d'une simple formation du sauveteur, ces associations de pratiques physiques organisent des exercicesexhibitions où l'on donne à voir - et à juger - des sauvetages ${ }^{52}$. Pour autant, aux yeux de la presse, seuls comptent l'acte de sauvetage et la bravoure de son auteur. La frontière sauveteur/sauveur est plus floue qu'on ne l'imagine.

À travers les chroniques du Nouvelliste du Morbihan relatant les actes de sauvetage, des valeurs morales émergent. Le sauveteur, parce qu'il est désintéressé, représente une morale en action. Le risque, la mise en danger de sa vie pour autrui, l'humilité et l'extraction souvent modeste du héros portent ses valeurs en lumière. Il symbolise aussi un espoir face à une destinée souvent fatale. De plus, le lecteur peut facilement s'identifier au sauveteur improvisé, un individu ordinaire qui, l'espace d'un instant, devient tout à fait extraordinaire. En plus de fournir un espoir et un exemple, le commun de ce sauveteur rend cet héroïsme accessible à n'importe quel individu. Toutefois, le traitement journalistique change en fonction du contexte de sauvetage, des caractéristiques de la victime et du statut du sauveteur. Si le canotier aguerri agit de façon collective, sécurisée et réfléchie, le sauveteur improvisé prend des risques réels qui mettent en péril sa vie autant qu'ils renforcent le versant impulsif de la décision. La nature profonde de cet homme y serait éminemment altruiste, ce qui correspondrait mieux à la notion de dévouement. La distinction dans le mode de narration relève de cette nuance majeure.

48. Thibaudeau, Patricia, Sauver des vies..., op. cit.

49. TREPARDoux, Francis, "Philippe-Nicolas Pia (1721-1799), échevin de Paris, pionnier du secourisme en faveur des noyés (première partie) ", Revue d'histoire de la pharmacie, $\mathrm{n}^{\circ} 315,1997$, p. 257-268.

50. LEVEAU, Philippe, "Évolution de la réanimation respiratoire vue à travers celle des noyés ", Histoire des sciences médicales, Tome 31, n 1, 1997.

51. CAILLE, Frédéric, "Le citoyen secoureur... ", op. cit.

52. VIVIER, Christian et RENAUD, Jean-Nicolas, " Les plaisirs nautiques au tournant des $19^{\mathrm{e}}$ et $20^{\mathrm{e}}$ siècles. Les mises en scène sportives organisées sur le Léman (Suisse) et sur le Doubs (France) ", Revue Loisir et société, automne 2010, p. 333-360. 


\section{Un traitement journalistique différent selon les contextes de sauvetage}

L'analyse des sources permet de distinguer les actes de sauvetage, non seulement au regard de l'aspect accidentel ou fautif à l'origine de la noyade, mais aussi selon qu'ils obéissent à une démarche anticipée ou spontanée couplée ou non à une rémunération de l'acte. La valorisation de ce geste de dévouement diffère et l'héroïsme afférent également.

\section{Lorsque survient l'improbable...}

Pour une noyade accidentelle, le journal classe les victimes selon trois types. Le premier concerne les victimes en activité professionnelle au moment des faits; le deuxième concerne les enfants qui tombent accidentellement dans l'eau. Le dernier se penche sur les adultes victimes d'un étourdissement ou d'un élément marin perturbateur (vague, courant violent) qui provoque l'immersion.

Pour le premier type de victimes, le récit de l'acte commence le plus souvent par l'identification de la victime : nom, prénom et parfois âge. Puis vient la description de son activité au moment de l'accident, tout en précisant sa profession et son lieu d'exercice. Le plus souvent un adjectif s'ajoute à la désignation ("La malheureuse ${ }^{53}$ ") afin d'en amplifier le caractère regrettable. Cette narration mobilise la compassion du lecteur envers cet infortuné, effectuant sa besogne quotidienne pour subvenir aux besoins de sa famille, et subissant les aléas impitoyables de l'océan. Le cœur de l'article s'applique, ensuite, à décrire l'acte de secours après avoir identifié le sauveteur selon le même rituel : nom, prénom, âge, adresse, profession et lieu d'exercice. Certains éléments symbolisent, aux yeux de l'anonyme journaliste, la force de l'acte et il s'agit de les mettre en valeur pour amplifier le sentiment de difficulté et de mise en danger. La profondeur de l'eau, la vitesse du courant mais aussi le nombre de fois où le sauveteur a dû plonger pour retrouver la victime apparaissent régulièrement ${ }^{54}$. Ces éléments préparent le lecteur. L'épilogue achève le processus de construction du héros providentiel. Le journaliste qualifie le bienfaiteur de courageux citoyen et le remercie pour son acte de dévouement. L'individu ordinaire se métamorphose, sous la plume du chroniqueur, en une incarnation idéalisée du citoyen républicain. La modernisation de la France et l'instauration progressive des valeurs de la III $^{\mathrm{e}}$ République transforment petit à petit le statut de chaque individu. Progressivement, l'incarnation de la puissance publique se sécularise. En miroir, le journaliste incarne de plus en plus le regard de la société pour celui qui en est le modèle.

53. "Sauvetage ", Le Nouvelliste du Morbihan du 15 mai 1887, p. 3.

54. OLIVIER, Vincent, "Un sang-froid et un courage au-dessus de leur âge ", Annales de Bretagne et des pays de l'Ouest, Tome 116, $\mathrm{n}^{\circ}$ 1, 2009, p. 73-85. 
Les lignes narrant le sauvetage d'un enfant mobilisent un canevas analogue, même si la chronique s'appesantit davantage sur la victime et sur les conséquences de l'accident. Le récit de l'acte se termine le plus souvent par le retour de l'infortuné(e) au domicile de ses parents. L'adresse alors précisée renvoie à l'idée que la responsabilité collective peut se substituer à celle des tuteurs légaux lorsque ceux-ci sont pris au dépourvu. L'accent est mis sur la compassion de la population témoin de l'accident et sur la détresse d'un enfant symbole d'innocence. Le journaliste remercie plus fortement le sauveteur d'avoir arraché le malheureux des griffes de la fatalité. L'Ankou est aveugle quant au choix de ses victimes.

Lorsqu'une lame ou une bourrasque vient happer la victime, les conditions météorologiques sont telles que l'acte prend des proportions épiques. Le sauvetage devient le combat titanesque de l'Homme face à la nature. Le sensationnel recherché par le journal pour emporter le lecteur dans le tourbillon enivrant des aventures quotidiennes trouve en la personne du sauveteur un héros identificatoire. L'entrefilet peut même se transformer en un article plus important, soutenu qu'il est par la puissance des détails.

En contexte accidentel, l'héroïsme est principalement lié à des facteurs qui accentuent la dangerosité de l'acte. " L'excellence civique courageuse n'appelle nulle évaluation abstraite ou philosophique; elle se mesure sur des risques, se compte à la force du courant, à l'heure de la journée, à la température, à la condition physique ou l'âge du méritant ${ }^{55}$ ". Ainsi, dans ce contexte imprévisible, le sauveteur représente en quelque sorte un protecteur des victimes contre un destin tragique guidé par la puissance invisible de la fatalité.

\section{Au bout de l'insoutenable...}

La narration des suicides reste relativement discrète. En effet, la morale demeure teintée des valeurs religieuses présentes dans les mentalités d'une société traditionnelle; elle censure les actes de mort volontaire qui contribuent à l'apparition d'un sentiment de culpabilité et ternissent la réputation d'une maisonnée ${ }^{56}$. La victime possède alors un statut particulier. Le plus souvent, le récit s'épanche sur la victime : l'endroit où elle vit, des faits concernant sa vie privée, tout en préservant régulièrement son anonymat :

«Vendredi dernier, $\mathrm{M}^{\mathrm{me}}$ Veuve D... demeurant à Vannes, rue des Orfèvres, qui avait perdu son mari depuis 4 mois, et donnait depuis cette époque des signes d'aliénation mentale, a tenté de se suicider vers cinq heures du soir, en se jetant à la mer, près la pointe d'Arradon. Un enfant qui se trouvait présent appela au secours. MM. Le Rohellec, père et fils, accoururent à ses cris et M. Le Rohellec, fils, sans prendre le temps de quitter ses effets, se jeta

55. CAILLE, Frédéric, La figure du sauveteur : Naissance d'un citoyen secoureur en France 1780-1914, Rennes, PUR, 2006, p. 183.

56. Glatigny, Jessica, " Une anticipation de l'inéluctable... ", op. cit. 
immédiatement à l'eau et fut assez heureux pour retirer la malheureuse que l'on put ramener à la vie à force de soins ${ }^{57}$."

L'article, en mentionnant ces mésaventures, semble faire preuve de compréhension à l'égard de la malheureuse : chagrin à la mort du conjoint, difficultés financières, abattement de malheurs successifs mais aussi " aliénation mentale ". Le journaliste cherche à faire comprendre le désespoir de son geste. Acculée par la vie, la personne n'avait pas d'autre choix. La fatalité reste présente mais émane de la personne et non de la mer. Ces procédés visant à expliquer le suicide permettent d'apaiser le sentiment de culpabilité éprouvé par l'entourage de la victime ${ }^{58}$. L'attention du lecteur est donc focalisée sur ces éléments de compréhension et d'explication de l'inexplicable; les sauveteurs passent au second plan. La société que ces derniers incarnent ne peut que s'adapter et les choix politiques de gestion du bord de mer ne sont aucunement égratignés, comme cela peut être sousentendu dans les situations accidentelles.

L'héroïsme apparaît ici avec moins de force que pour le sauvetage d'un enfant ou d'un adulte accidenté. Les détails de la vie privée de la victime phagocytent le propos. Or, la générosité de l'acte serait dépendante de la valeur " humaine " de la victime, expliquant l'atténuation de l'héroïsme qui découle du récit d'un suicide par noyade. Ces chroniques taisent toute compassion. Vécue comme une injustice pour de nombreux marins et leur famille, la noyade volontaire serait incompréhensible, dénigrée, inconcevable et immorale. Plus encore, ces sauvetages ne peuvent mobiliser que des héros providentiels puisque la personne suicidaire ne peut être identifiée comme telle qu'à travers les témoignages d'autres citoyens, souvent à terre, mais aussi en raison de l'anomie sous-jacente qui pousse l'intéressé(e) à éviter les lieux fréquentés, surveillés par des professionnels.

\section{Nul n'est infaillible...}

Certaines victimes commettent des erreurs de navigation ou des fausses manœuvres, souvent plus au large. D'autres chutent dans l'eau, pris de boisson. Dans ce contexte, la plupart des articles font preuve d'une morale manichéenne. Le bien est incarné par le sauveteur et le mal est représenté par l'individu qui se met en danger. La fatalité s'esquive au profit d'une valorisation de l'ordre moral. À la suite d'une action inconsidérée, le sauveteur répare en quelque sorte la mauvaise action; il sauve cette victime, fautive auprès de son héros comme auprès de la communauté qu'elle a abusée.

Le premier type de scénario marque bien cette logique d'émergence de l'individu comme responsable de ses actes. La répartition dualiste des rôles s'accroît lorsque l'erreur de manœuvre cause le décès de l'un des personnages. Les conditions de l'accident sont alors très détaillées, afin

57. « Tentative de suicide », Le Nouvelliste du Morbihan du $1^{\text {er }}$ mai 1887.

58. GlatignY, Jessica, "Une anticipation de l'inéluctable... », op. cit. 
de mettre en évidence l'inexpérience du responsable du naufrage et son incapacité à réagir de manière adaptée. Le discours protège et valorise du même coup le reste de la corporation en isolant le fautif. Le chroniqueur accuse la personne incriminée de la " perte de son sang froid ${ }^{59}$ ". Son manque de cran le rend coupable du sort de la victime et relègue l'acte de sauvetage, désespéré et inefficace, au second plan. L'héroïsme serait une fête irrémédiablement gâchée par la tristesse et la compassion que le décès génère. Lorsqu'il s'agit d'un enfant, la tristesse et le désarroi de la mère se conjuguent à ceux de la population et les effets tragiques sont décuplés :

"Au premier rang à côté de nous se trouvait la mère de l'enfant. Il est impossible de dépeindre la désolation de la malheureuse mère, qui n'avait connu le malheur qui la frappait qu'en recevant dans ses bras le cadavre du pauvre petit ${ }^{60}$."

Le journaliste joue sur la proximité physique ("à côté de nous ") pour induire une proximité affective d'où l'empathie naîtra.

A contrario, sans victime, les pertes matérielles concentrent l'attention du journaliste et éclipsent l'activité du sauveteur sans toutefois l'occulter. Nom, prénom, âge, profession et, parfois le lieu d'exercice, restent les informations minimales suffisantes à la gratification symbolique de son geste impétueux. Mais, une fois de plus, le centre de gravité de la chronique porte sur l'erreur, la " faute originelle ", des marins. La mise en danger de son existence pour porter secours aux naufragés concourt effectivement à la construction de l'héroïsme quotidien, mais face à la fatalité, le développement d'un comportement modèle peine à s'opposer à l'imaginaire des " risques du métier ". La culpabilisation du fautif l'emporte sur le droit à l'erreur dans une société implacable.

Le même procédé œuvre dans le cas des chutes pour abus de boisson. Un homme en état d'ébriété, qui tombe dans le port, porte alors des qualificatifs peu reluisants. Pourtant, dans une société qui lutte contre le fléau de l'alcoolisme dans une démarche sanitaire autant qu'hygiéniste, le buveur breton bénéficie d'une certaine magnanimité. Cette mise à l'index de la victime responsable de son propre malheur reste d'autant plus présente dans le journal que la logique républicaine bat en brèche les postures déterministes et vante les succès de chacun. Toute personne est maintenant détentrice de son destin et, à ce titre, responsable. L'article est souvent court et le sauvetage est peu détaillé, le sauveteur quant à lui reste nommé mais la description de son acte reste purement factuelle.

Les accidents impliquent donc la responsabilité d'un citoyen. La description de l'erreur prédomine alors toujours et réduit la description de l'acte de sauvetage à sa portion congrue. L'héroïsme est moins fortement mis en avant dès lors qu'il n'exprime pas le combat qu'il représente contre

59. "Un accident dans la rivière du Scorf ", Le Nouvelliste du Morbihan du 2 juin 1887, p. 2.

60. Ibidem 
une force supérieure, celle de la fatalité et/ou de la nature. Dans sa relation à sa communauté, l'individu fautif est mis en défaut. L'illustration de la puissance de la chose publique comme barrière aux influences malheureuses de la fatalité semblerait mise à mal par le comportement anomique de certains individus. Le Nouvelliste du Morbihan ne promeut donc pas ces situations.

\section{L'avènement des loisirs...}

Baigneurs, parfois baigneuses, navigateurs de plaisance et pêcheurs d'agrément investissent les rivages. La villégiature du bord de mer touche, au-delà des falaises biarrotes sous Napoléon III, les criques morbihannaises. Les imprudents d'alors négligent parfois les aléas de l'océan.

« Tout à coup, le jeune Salen disparut dans un trou et comme il ne savait pas nager, il allait infailliblement se noyer quand le jeune Émile Lyncey qui s'était déjà habillé, n'écoutant que son courage, se jeta tel quel à son secours et put ramener le malheureux enfant complètement évanoui sur la rive ${ }^{61}$."

Pourtant, le discours journalistique se rapproche du contexte d'erreur au point de rendre la distinction peu évidente. La plupart des victimes sont emportées par le courant, submergées par une vague ou simplement prises de panique lorsque le niveau de la mer les déborde. Une responsabilisation du baigneur qui se met en danger est présente; la morale instillée dans ce journal a tendance à condamner l'oisiveté. Le baigneur réalise une action purement centrée sur lui, ce qui est contraire à l'action du sauveteur, qui met sa vie en péril pour sauver celle d'un autre. Pour autant, les adeptes de ces pratiques sanitaires ne peuvent être assimilés aux fautifs de naguère. Cette strate souvent aisée de la population façonne quelque part la société à son image ${ }^{62}$, elle ne saurait incarner la légèreté, l'irresponsabilité ou l'immoralité. Le propos serait alors affecté par la représentation positive que les journalistes comme la société ont des personnes concernées. De plus, la proximité de la mer rend la baignade facile.

Dans ce type d'article, une importante attention est attachée à la description de l'acte de sauvetage. Les détails fournis par le journaliste, semblables à ceux utilisés dans les situations accidentelles, accentuent pareillement la bravoure du généreux plongeur : force du courant, profondeur de l'eau, etc. La trame empruntée pour la description commence toujours par une identification du rescapé ainsi que du lieu de l'incident. Le décor planté, le chroniqueur explique ce qui a perturbé la quiétude initiale pour conduire à la noyade. Puis, le film de l'article se poursuit avec l'entrée en scène du sauveteur; son analyse de la situation, son action. Cette chrono-

61. "Sauvetages ", Le Nouvelliste du Morbihan du 28 août 1892, p. 2. Le "infailliblement " est mis en gras par nous.

62. HalBWACHS, Maurice, Les classes sociales, Paris, Éditions Tournier et Constant, 1942, cité dans DAVIET, Jean-Pierre, La société industrielle en France 1814-1914, Paris, Seuil, 1997, p. 23. 
logie accroît la dynamique potentiellement dramatique de l'acte de dévouement dans la mesure où l'individu qui s'improvise sauveteur ne marque aucune hésitation pour porter secours, malgré les dangers qu'offre autant le milieu marin qu'une personne paniquée, guidée par ses instincts de survie. L'individu ordinaire devient extraordinaire à partir du moment où il risque sa vie. Il s'ensuit une description du sauvetage lui-même où chaque détail amplifie l'action du sauveteur puis, lorsque la victime est hors de danger, l'article se termine par une phrase de remerciement à " ce brave citoyen $^{63}$ " avec parfois un rappel de ses sauvetages précédents.

La noyade chez le baigneur aisé n'est aucunement une faute et rejoint le mode de désignation d'un sauvetage en situation accidentelle. Cette strate de la société ne saurait défaillir. Elle devient donc support à valorisation d'un honnête homme de modeste extraction devenu, le temps du sauvetage, l'incarnation idéalisée du solidarisme de la III $^{\mathrm{e}}$ République. La valeur morale du généreux nageur rehausse sa qualité par son geste.

\section{La gestion du probable ou le sauveteur professionnel}

Les récits de sauvetages improvisés du Nouvelliste du Morbihan suivent une structure récurrente. Un sauveteur, n'écoutant que son courage, met en péril sa vie pour sauver celle d'autrui. L'acte auréole un individu en particulier et s'étend, par son anonymat, sur tous les concitoyens. Paradoxalement, les sociétés spécialisées ne bénéficient assurément pas du même traitement. Elles n'apparaissent la plupart du temps que dans le cadre d'articles relatant les obtentions de subventions accordées à ces associations par l'État, le clergé ou des entreprises de commerce maritime. Les bonnes actions qu'elles ont accomplies sont certes récompensées par des dons de la part des citoyens selon le mode philanthropique connu, mais elles ne bénéficient pas de la même visibilité journalistique. Les remises de récompenses sont également décrites. Des propos élogieux saluent l'action d'un sauveteur ou d'un équipage de canot avec, parfois, un bref rappel des sauvetages précédents (le nom du bateau abîmé en mer ou le nom de la victime). Toutefois, l'analyse des colonnes du Nouvelliste ne permet pas de lire un récit de sauvetage par un canot de sauvetage portant secours à un équipage d'un navire naufragé. Il semblerait que le bihebdomadaire donne symboliquement au sauveteur improvisé ce que la population donne de manière pécuniaire à la Société. Au-delà d'une possible compensation symbolique, il est vrai que les chroniqueurs peuvent plus facilement trouver des témoignages sur terre qu'en haute mer, à défaut d'être présents eux-mêmes sur le lieu des naufrages et des noyades. Par ailleurs, si la prise de risque et la mise en danger de sa propre existence semblent être une condition sine qua non pour définir un acte de sauvetage digne de ce nom, la rationalisation des techniques d'intervention par une recherche de sécurité du sauveteur lors son intervention transforme le rap-

63. «Sauvetage ", Le Nouvelliste du Morbihan du 15 mai 1887, p. 3 
port à l'idéal. Le canot groisillon " l'Amiral-Mecquet " (1865-1901), équipé de caisses à air dans un but de flottabilité accrue ${ }^{64}$, en est un parfait exemple. L'équipement évolue. Le gilet fait son apparition. Les marins connaissent davantage les côtes, les vents, les courants et les embûches de l'océan. La confection d'outils adaptés (filins, ancres flottantes, etc.) et l'amélioration des canots de sauvetage, afin de les rendre insubmersibles, participent finalement de la réduction des qualités intrinsèques de l'individu pour valoriser une démarche plus professionnelle et rationnelle. Mais cette dernière rend l'identification plus délicate. Ces évolutions expliquent largement la distinction de traitement et n'empêche nullement de questionner plus globalement la place du sauveteur anonyme dans Le Nouvelliste du Morbihan.

L'acte du héros providentiel se joue des risques pris par ce dernier. Au plan social, la description réunit en une même histoire la proximité et son humilité. Le sauveteur de la société de sauvetage apparaît dans les colonnes de la presse à l'occasion, moins spectaculaire, d'une remise de récompense, de titre honorifique ${ }^{65}$ ou de subvention ${ }^{66}$, lors de la présentation d'un nouveau matériau de sauvetage ${ }^{67}$ voire de l'assemblée générale ${ }^{68}$. C'est un groupe et non une icône qui est mis en avant.

Le pragmatisme et la réduction des risques dus aux progrès techniques contrarient la logique sensationnaliste de la presse. Si les sociétés spécialisées cherchent auprès des édiles les reconnaissances financières nécessaires à leur bon fonctionnement, le bihebdomadaire porte son attention sur un individu qui n'a comme médaille à son revers que la reconnaissance invisible de la population à son endroit. Le développement du sauvetage en équipage induit également une rationalisation de l'acte et la vie du sauveteur devient plus importante que celle du sauvé. Au plan professionnel, les secours ont besoin de bras qualifiés, non de héros... morts.

\section{Tableau récapitulatif de la présence du sauveteur professionnel dans Le Nouvelliste du Morbihan}

\begin{tabular}{|c|c|c|c|c|c|c|}
\hline & 1887 & 1892 & 1897 & 1903 & 1907 & 1914 \\
\hline Improvisé & 27 & 35 & 30 & 22 & 28 & 15 \\
\hline Professionnel & 4 & 4 & 7 & 2 & 3 & 5 \\
\hline Total & 31 & 39 & 37 & 24 & 31 & 20 \\
\hline
\end{tabular}

Dans ces conditions, si le nombre d'interventions fluctue autour d'une trentaine par an sur la période étudiée, aux impondérables près, le rapport

64. TONNERRE, Laurence et Guy, Groix, L'île des sauveteurs, Spézet, Coop Breizh, 2003.

65. «Hospitaliers Sauveteurs Bretons », Le Nouvelliste du Morbihan du 16 juin 1887 p. 1.

66. " Pour les Hospitaliers Sauveteurs Bretons ", Le Nouvelliste du Morbihan du 27 mai 1897, p. 1.

67. " Appareil de Sauvetages ", Le Nouvelliste du Morbihan du 4 juillet 1897, p. 1.

68. "Société centrale de sauvetage ", Le Nouvelliste du Morbihan du 15 mai 1887, p. 1. 
entre intervention du héros providentiel face au héros ayant déjà fait ses preuves ${ }^{69}$ reste grandement en faveur de l'anonyme sauveteur. Par-delà l'effet de source qui conduit à sur-valoriser l'activité visible au plan physique (sauvetage dans le port par rapport au sauvetage en haute mer), le média de la presse locale semble véritablement mettre en lumière un héros quotidien, modeste et généreux dont les valeurs en action semblent satisfaire celles de la III ${ }^{\mathrm{e}}$ République, sans pour autant froisser celles d'une religion encore prégnante dans la région. Le discours journalistique s'appuie sur une qualification en adéquation avec un idéal républicain identifié qui s’impose comme un modèle accessible fondé sur le dévouement.

À travers Le Nouvelliste du Morbihan, les valeurs individuelles s'insèrent dans celles d'une société en cours d'élaboration et des héros ordinaires peuvent incarner la nouvelle norme sociale. L'exemplarité du sauveteur est soulignée régulièrement dans la presse locale de la Belle Époque. Pardelà les actes de probité et de bravoure communs (portefeuille retrouvé avec des sommes importantes en leur sein, intervention opportune devant une voiture de foin qui allait écraser un enfant, gestion courageuse d'une monture mettant en danger son maître, etc.), la récurrence de cette figure le prouve mais les sauvetages aquatiques prennent une dimension supplémentaire. La noyade signifie généralement la mort. Son statut, auprès d'un lectorat de bord de mer, surclasse les autres natures d'incident. Le mode d'identification y fonctionne effectivement plus aisément. Malgré l'attachement de l'espace breton aux croyances religieuses, le citoyen s'impose doucement, par voie de presse, comme capable de s'opposer aux forces du destin, jusqu'alors divines. Toutefois les valeurs que symbolise le sauvetage restent identifiables malgré la présence d'artifices, dans la mesure où une logique philanthrope reste prédominante dans tout type d'acte de sauvetage. Plus encore que la philanthropie, nous percevons derrière l'idéal démocratique les résistances à l'égalitarisme, puisque les classes aisées restent distinctes de celles plus plébéiennes. Cependant, la solidarité à l'œuvre dans ces instants vitaux permet de saisir une sécularisation progressive de la société par une réduction de la part du divin dans l'écriture de la vie des hommes.

L'acte de sauvetage dans la presse devient donc le théâtre de l'expression d'une grandeur civique qui reste symbolique. Il est assez courant de rencontrer dans les pages du journal un sauveteur qui souhaite garder son anonymat et refuse toute distinction institutionnelle. Ce phénomène donne donc l'image d'un individu prêt à se dévouer de manière totalement désintéressée. Cette humilité du sauveteur apporte au statut quelque chose de particulier, qui embellit dans l'esprit du lecteur son héroïsme. Le sauveteur entretient donc l'image d'un individu totalement dévoué à autrui

69. Modèle initial de Cincinnatus selon GIRARDET, Raoul, Mythes et mythologies..., op. cit., p. 72. 
sans attente en retour; il s'ensuit une forte reconnaissance symbolique de la part de la population. Il semble qu'un enracinement de l'image du sauveteur en tant que héros prend forme dans l'imaginaire d'une société sous la III République, dans les colonnes de cette presse locale devenue la première de la ville pendant notre période. Les sauveurs jusqu'alors incarnés par des soldats ayant payé de leur sang le prix de leur don à la collectivité sont remplacés, dans une période pacifiée, par ces soldats du quotidien payant de leur peur l'honneur, modeste mais visible, que la presse récupère et imprime.

L'analyse des actes de sauvetage relatés dans Le Nouvelliste du Morbihan permet de déceler une construction d'un héroïsme d'un genre nouveau, plus commun, plus normal, celui d'un soldat du quotidien. Entre 1887 et 1914, le filtre des valeurs morales fait résonner une idéologie républicaine et citoyenne en devenir. L'héroïsme, fondé sur la notion de dévouement, implique une prise de risque pour la vie du sauveteur et il est d'autant plus tangible qu'il est providentiel et désintéressé. Mais la finesse du discours journalistique consiste à valoriser cette attitude de manière mesurée selon les conditions dans lesquelles l'événement s'est produit. Face à la fatalité d'une mer aveugle, l'Homme prend toute sa dimension. Son acte s'en trouve renforcé. Quand cet aléa de l'existence tente de faucher un enfant, la valeur de son geste redouble. Si la situation résulte d'un comportement coupable, la société jette son opprobre et l'inculpation réduit la valorisation du sauveteur. Bref, on ne vaut qu'à travers ce que l'on sauve : une classe sociale aisée, balnéaire ou un honnête travailleur d'un côté et l'alcoolique ou le suicidaire de l'autre. Cette étude permet de saisir comment, lorsque les valeurs de la III ${ }^{\text {e }}$ République s'invitent dans les trajectoires individuelles, un même acte peut mettre en lumière les tensions qui habitent un espace social ayant la grève pour frontière. La structure et la cohésion des sociétés de sauvetage génèrent une visibilité plus stable, moins exceptionnelle, moins vendeuse. Par ailleurs, si l'héroïsme trouve, dans un acte spontané, la force et la vertu de l'exemple moral, il convient également d'en concevoir les limites. La mise en scène du geste altruiste du sauveteur change radicalement lorsque son auteur appartient à un groupe entraîné. Le poids de la collectivité l'emporte sur l'exemplarité singulière.

Entre cohésion sociale et méritocratie, l'acte de sauvetage représenté dans la presse semble prendre toutes les nuances possibles, justifiant les silences et les articles prolixes, les dénonciations et les excuses. Les feuillets locaux s'imposent dès lors sûrement comme des relais sub-politiques ${ }^{70}$ efficaces dans la diffusion de normes.

70. BECK, Ulrich, La société du risque. Sur la voie d'une autre modernité, Paris, Flammarion, 2000, p. 400. 


\section{RÉSUMÉ}

L'étude du Nouvelliste du Morbihan sur la période de 1887 à 1914 permet l'analyse de la construction de la figure du héros providentiel développé à travers les récits des actes de sauvetage dans la région de Lorient. Ce héros est fortement marqué par des valeurs provenant d'une troisième République naissante. En outre, le récit des luttes contre l'un des grands fléaux des zones portuaires, la noyade, semble être un moyen efficace de promouvoir une idéologie citoyenne. Ce journal présente le héros ordinaire comme une figure exemplaire faisant preuve d'acte de dévouement. Ainsi, deux profils de sauveteurs émergent. Dans le premier profil, le risque est au cœur du sauvetage et c'est pourquoi il est plus propice au spectaculaire et au sensationnel, ce qui est vivement apprécié et recherché par le journaliste. Le second profil est traité différemment par le chroniqueur. En effet, le sauveteur professionnel, par son équipement et sa connaissance des stratégies de sauvetage, tend à diminuer la prise de risque. Par conséquent, le sauvetage est moins spectaculaire et moins à même d'être présenté comme un acte héroïque par le journaliste.

\section{ABSTRACT}

This study of the Nouvelliste du Morbihan, in the years between 1887 and 1914 highlights how the figure of the providential hero developed throughout narratives of rescue acts in the Lorient area. This hero embodied the values of the newly born Third Republic. Moreover, the fight against one of the major scourges of port area, drowning, is an efficient way of promoting an ideological agenda. The newspaper characterises the ordinary hero as an exemplary figure imbued with a devotion to duty. Two profiles of rescuers emerged from this analysis of the Nouvelliste du Morbihan. In the first profile, risk was at the heart of the rescue and that is why it was more spectacular and sensational - a behaviour appreciated by journalists. The journalists treated the second profile differently. Indeed, the professional rescuer was equipped and possessed a rescue strategy that decreased risks during the rescue. Therefore, the rescue fact was less spectacular and less suitable to be presented as an epic act by the journalist. 